\title{
2017-2018 Reviewers-Consultants 2017-2018
}

The following individuals have graciously provided reviews for the Canadian Journal of Experimental Psychology during the period of November 2017 to November 2018. We thank them for their thoughtful and timely assistance.

De novembre 2017 a' novembre 2018, les personnes suivantes ont offert leur collaboration spéciale dans l'évaluation de manuscrits pour la Revue canadienne de psychologie expérimentale. Nous les remercions de leur aide précieuse.

Scott Allen

Jackie Andrade

Katherine Arbuthnott

Mark Ashcraft

Maria Augustinova

Tanjeem Azad

Joe Baranski

Juan Botella

Valerie Camos

Michel-Pierre Coll

Lola Cuddy

Evan Curtis

Sarah D'Amour

Alain Desrochers

Digby Elliott

Michael Fartoukh

Jonathan M. Fawcett

Shirley Fecteau

Christian Frings
Winston Goh
Marianne Habib
Alan Hartley
Mary Hegarty
William E. Hockley
Mark J. Huff
Rob Hughes
Karin Humphreys
Frédéric Huppé-Gourgues
Zahra Hussain
Tom James
Brendan Johns
Raymond Klein
Ethan Kutlu
Anne Lafay
Jason P. Leboe-McGowan
Jakob Leimgruber

Zhong-xu Liu

Stephen Lupker

Erin Maloney

Jonathan Marotta

Leonie M. Miller

Bruce Milliken

Toby Mordkoff

Jason D. Ozubko

Pauline Pearson

Jean-Charles Pelland

Gordon Pennycook

Manuel Perea

Jerome Prado

Katherine M. Robinson

Steven Roodenrys

Kathrin Rothermich

Annie Roy-Charland

Christopher Sears
Monique Sénéchal

Robert Siegler

Alex Taikh

Chris Taylor

Gerry Tehan

Helen Tibboel

Elise Tornare

Nash Unsworth

Francois Vachon

Jeffrey D. Wammes

Steven Weisberg

Veronica Whitford

Doug Williams

Julia Xing

Regine Zopf 